Horizontes. Revista de Investigación en Ciencias de la Educación

ISSN 2616-7964

octubre-diciembre, 2017

Volumen 1, Número 4

pp. 16-27

www.revistahorizontes.org

(c) (i) (S) (2)

\title{
Propuesta de un programa de formación educativo- jurídico para la sensibilización de la población campesina del municipio Paz Castillo del estado Miranda en la defensa de su derecho a la tierra
}

\section{Proposal of an educational-legal training program for the sensitization of the rural population of the Paz Castillo municipality of the Miranda state in the defense of their right to land}

\author{
Judith González \\ jjgonzacolon@gmail.com \\ Universidad Nacional Abierta \\ Universidad Bolivariana de Venezuela
}

Recibido: 09-04-2017 / Revisado: 14-04-2017 / Aceptado: 17-05-2017 / Publicado: 09-10-2017

\begin{abstract}
RESUMEN
Este trabajo de investigación tiene como objetivo programa de formación educativo-jurídico para la sensibilización de la población campesina del municipio paz castillo del estado Miranda en la defensa de su derecho a la tierra, el interés de realizar esta investigación nace en virtud que, la formación continua constituye la principal herramienta para asegurar y garantizar el goce efectivo de los derechos humanos. Los basamentos teóricos son Gilbert (2013) autor que plantea que el derecho a la tierra constituye un derecho humano fundamental, ya que está asociado a derechos de supervivencia como el derecho a la alimentación. Metodológicamente es un proyecto factible siguiendo a la UPEL (2006), con apoyo de una investigación de campo, se aplicó una encuesta a una muestra de sesenta (60) sujetos de investigación. El análisis de resultados permitió constatar que la muestra seleccionada desconoce los mecanismos del Estado para garantiza los derechos humanos. Sin embargo, es necesaria la continua formación de todos como garantes de las leyes. Se recomienda estimular el proceso de formación y aprendizaje como estrategia primordial en el cumplimiento de los derechos humanos que fortalezca las instituciones garantes de su goce efectivo.
\end{abstract}

Palabras clave: Derechos humanos, Derecho a la tierra, Formación

\begin{abstract}
This research work aims to educational-legal training program for raising awareness among the rural population of the municipality of Castillo de Miranda state in the defense of their right to land, the interest of carrying out this research is born in virtue of the fact that continuous is the main tool to ensure and ensure the effective enjoyment of human rights. The theoretical foundations are Gilbert (2013) author who states that the right to land is a fundamental human right, since it is associated with rights of survival such as the right to food. Methodologically it is a feasible project following the UPEL (2006), with the support of a field investigation, a survey was applied to a sample of sixty (60) research subjects. The analysis of results allowed to verify that the selected sample does not know the mechanisms of the State to guarantee the human rights. However, the continuous formation of all as guarantors of the laws is necessary. It is recommended to stimulate the process of training and learning as a fundamental strategy in the fulfillment of human rights that strengthens the institutions guaranteeing their effective enjoyment.
\end{abstract}

Key words: Human rights, Right to land, Training 


\section{INTRODUCCIÓN}

El reconocimiento del derecho a la tierra como derecho humano, representa la reivindicación histórica de los movimientos campesinos, dada la importancia histórica que en este momento el acceso a la tierra para la seguridad alimentaria y la realización del derecho a la alimentación, la conservación de la biodiversidad y la lucha contra el cambio climático. Normalmente, el derecho a la tierra no es concebido como derecho humano; sin embargo, se trata de un tema esencial; ya que, tiene un impacto real sobre el disfrute del derecho a la alimentación, a la vivienda, a la salud, al trabajo, a un medio ambiente saludable, al desarrollo $\mathrm{y}$, sin acceso a la tierra, muchos pueblos o comunidades están privados de sus medios de subsistencia.

En la actualidad un número significativo de agricultores no poseen títulos de propiedad, lo cual implica una minusvalía en el ejercicio de los derechos al no poder acceder a financiamientos otorgados por las instituciones bancarias que les permita optimizar el desarrollo de actividades agropecuarias. De esta realidad, no escapa la región de la los Valles del Tuy, población rural del estado Miranda perteneciente al municipio Paz Castillo, zona cuya principal actividad económica es la agricultura y que constituye uno de los 21 municipios que integran el estado. Región en la cual se hace necesaria la incorporación de estrategias que contribuyan a la sensibilización para la defensa del derecho a la tierra.

En este contexto, se plantea el presente proyecto el cual tiene como propósito proponer un programa de formación educativo - jurídico para la sensibilización de la población campesina del Municipio Paz Castillo del estado Miranda en la defensa de su derecho a la tierra. La investigación reviste de singular importancia, porque contribuirá con la sensibilización de la población campesina del Municipio Paz Castillo del estado Miranda en la defensa del derecho a la tierra. Asimismo, se transformará en una contribución al estudio del derecho a la tierra, tema vinculado estrechamente con la garantía de un conjunto de derechos humanos, en especial el derecho a la alimentación.
El acceso a la tierra tiene una importancia vital porque se trata de un activo esencial para la producción de alimentos y de un factor básico desde el punto de vista de la vivienda y el desarrollo comunitario. En consecuencia, las normas que lo regulan son de rango constitucional, su aplicación es de forma inmediata, directa y preferente por los órganos jurisdiccionales y demás órganos del poder público.

La propiedad de la tierra no es un bien como otros, ya que está asociado con el derecho a la alimentación, un derecho humano que incide en el goce efectivo del resto de los derechos, vinculado estrechamente con la lucha de la pobreza y la promoción de mejores condiciones para el desarrollo rural integral. En consecuencia, mantener la tierra con vocación agrícola ociosa y la concentración en pocas manos, atenta contra el desarrollo agroalimentario del país, así como con el uso óptimo de la tierra. También constituye una violación al derecho a la propiedad que tiene la población agrícola que labora la tierra, limitando la generación de empleo y por ende, a un nivel adecuado de bienestar.

En el marco de las observaciones anteriores, la Organización de las Naciones Unidas para la Alimentación (FAO) (2014), señala que:

El derecho a la tierra y la estabilidad de ese derecho en el tiempo incide significativamente en el desarrollo económico, por ende, el Estado debe reconocer los derechos individuales sobre la tierra, así como generar mecanismos legales $\mathrm{u}$ otras formas institucionales para defender esos derechos, sin incurrir en costos prohibitivos. (p.27)

La Declaración de la Organización de Naciones Unidas (ONU) sobre el Progreso y el Desarrollo (1969), establece la utilización de la tierra en interés de la sociedad como medida necesaria para el desarrollo y el progreso, y la reforma agraria como mecanismo para hacer del derecho a la propiedad una herramienta fundamental en la justicia social y el desarrollo económico. Igualmente, la Declaración de Vancouver sobre Asentamientos Humanos (1976), establece que la existencia de un sistema de 
leyes sobre tenencia de la tierra es fundamental para asegurar la realización de los objetivos básicos de la reforma social.

El derecho a la propiedad de la tierra cumple la función social de garantizar la seguridad alimentaria del país; en consecuencia, está estrechamente vinculado al ejercicio del derecho a la alimentación. Provea (2016) señala que, a pesar de la magnitud de recursos financieros gubernamentales y del crédito destinado por la banca pública y privada para aumentar la producción nacional, Venezuela continúa siendo un importador neto de alimentos. Igualmente, entre los problemas que mayormente afecta a la población campesina, es la falta de titularidad de las tierras.

En la actualidad un número significativo de productores agrícolas sólo posee títulos de adjudicación como único instrumento legal para demostrar la posesión de la tierra que trabajan, lo cual implica una minusvalía en el ejercicio de los derechos al no poder acceder a financiamientos otorgados por las instituciones bancarias que les permita optimizar el desarrollo de actividades agropecuarias, como tampoco impedir las continuas invasiones, saqueos a las cosechas y persecuciones de grupos armados de las que son víctimas. De acuerdo con Guarenas (2011):

En Venezuela al menos 256 dirigentes campesinos líderes de las luchas por el derecho a la tierra, han perdido la vida en los últimos ocho años producto del accionar del sicariato, a pesar de los reiterados reclamos realizados por diversas organizaciones campesinas y de derechos humanos. (p.23)

Aunado a, los constantes hechos violentos que se desarrollan en las poblaciones rurales, consecuencia de las luchas por la propiedad de la tierra. Por otra parte, si bien es cierto que el Estado venezolano promulgó un conjunto de leyes dirigidas a garantizar el derecho de la propiedad de la tierra; así como también, creó un conjunto de instituciones responsables del diseño y ejecución de las políticas públicas agrarias para fortalecer el sector agrícola; entre ellas el otorgamiento de los títulos de propiedad o adjudicación de terrenos; sin embargo, estas acciones ha sido poco efectivas en el logro de los objetivos de fomentar, respetar, proteger y cumplir el derecho a la tierra; por el contrario las políticas agrarias socavan la seguridad de la propiedad de los pequeños agricultores, como se evidencia en las constantes denuncias sobre los vicios en el otorgamiento de la titularidad de la tierra.

De esta realidad, no escapa la región de la los Valles del Tuy, población rural del estado Miranda perteneciente al municipio Paz Castillo, zona cuya principal actividad económica es la agricultura y que constituye uno de los 21 municipios que integran el estado. Posee una superficie de $408 \mathrm{~km}^{2} \mathrm{y}$, de acuerdo con el INE, su población es de 112.357 habitantes.

La población rural del municipio $\mathrm{Paz}$ Castillo constantemente es expuesta a la ausencia de políticas públicas que garanticen el ejercicio y goce efectivo de sus derechos, en especial el derecho a la tierra. Para García (2016), los Valles del Tuy es una zona principalmente productora agrícola; sin embargo, las condiciones de deterioro de las vías, de inseguridad $\mathrm{y}$, en especial la falta de títulos de propiedad, impiden mantener el desarrollo de esta actividad económica.

Adicionalmente, el autor antes mencionado, afirma que la redistribución de la tierra continúa siendo una cuestión crítica en el sector de los Valles del Tuy; pues ha sido una de las zonas preferidas para las construcciones de complejos habitacionales, potenciado en la actualidad por la dinámica de la Gran Misión Vivienda. Según cifras de Corpomiranda (2016), indican que en los Valles del Tuy han entregado más de 45.000 viviendas desde 2012 hasta la fecha. Esta situación ha contribuido a generar el interés en sectores sociales a influir en el cambio de la vocación de la tierra de agrícola a urbana. Estas tierras tampoco estaban necesariamente desocupadas o sin uso, ya que en una parte importante de ellas había ocupantes que poseían las tierras, que contaban con una tenencia precaria y con bienhechurías. 
En la defensa y protección del ejercicio y goce efectivo del derecho a la tierra, es fundamental la incorporación activa de los sujetos de este derecho; es decir, los campesinos. Al respecto, la Ley de Tierras y Desarrollo Agrario establece como propósito el desarrollo rural integral en términos de productividad de la tierra; por tal razón, son sujetos de aplicación de la misma, los productores agropecuarios. Son sujetos beneficiarios del régimen establecido en esta Ley, todos los venezolanos que hayan optado por el trabajo rural, especialmente para la producción agrícola y el desarrollo agrario, como oficio u ocupación principal.

Sin embargo, la población de los Valles del Tuy progresivamente se está transformando en la extensión de la ciudad capital, perdiendo con ello el interés por la producción agrícola, transformándose en consumidores y no en productores agrícolas y por ende, por la lucha por el derecho a la propiedad a la tierra situación que se evidencia en la casi inexistencia de organizaciones campesinas en la región, así como la incorporación de la población a hechos delictivos. Por otra parte, la distribución desigual de la tierra y el crecimiento de población en la región, ha conllevado que los productores agrícolas subdividan sus parcelas entre los miembros de la familia, lo que provoca una marcada reducción en la relación tierra/persona. Adicionalmente, la ineficacia del Estado para dar la titularidad del derecho, ha fomentado las invasiones, situación que ha sido una constante en la región de los Valles del Tuy.

En este contexto, se hace necesaria la incorporación de estrategias que contribuyan a la sensibilización de la población campesina de los Valles del Tuy, en especial del Municipio Paz Castillo del estado Miranda, mediante la educación en y para los derechos humanos, tema de una singular vigencia, dado los cambios culturales que han incidido significativamente en los valores que la actual sociedad venezolana demuestra, y la relación existente entre éstos y los derechos humanos. La educación constituye una herramienta que facilita la formación de ciudadanos sujetos plenos de derechos y la construcción de una ciudadanía capaz de defender y promocionar los derechos humanos.

Freire (1971) señala que desde la concepción, los sistemas de educación no formales proporcionan las bases necesarias para el desarrollo cultural de las personas, genera nuevos horizontes y rompe barreras histórico-culturales. Para el autor antes mencionado, la educación no formal se refiere a las actividades realizadas fuera del ámbito escolar, con el propósito de desarrollar competencias y facultades intelectuales $y$ morales de los individuos. Según el autor, el proceso educativo tiene como propósito facilitar el aprendizaje, siendo la educación un hecho práctico y reflexivo, que realiza el hombre sobre el mundo para transformarlo. Para el autor, la educación no es un hecho aislado de la realidad social, por lo que ésta la influye y la determina.

En este contexto, se plantea como Objetivo de la Investigación proponer un Programa de formación educativo - jurídico para la sensibilización de la población campesina del Municipio Paz Castillo del estado Miranda en la defensa de su derecho a la tierra.

\section{DISCUSIÓN Y RESULTADOS}

En esta sección de la investigación se realizó un diagnóstico sobre las $\mathrm{E}$ a la encuesta aplicada al total de la muestra seleccionada sobre los conocimientos sobre la eficacia de las políticas públicas y los mecanismos establecidos por el Estado venezolano para la garantía de su derecho a la tierra. En tal sentido se puede señalar que no poseen conocimientos, lo cual puede observarse en el Gráfico $\mathrm{N}^{\circ} 1$ y Tabla $\mathrm{N}^{\circ} 2$. 


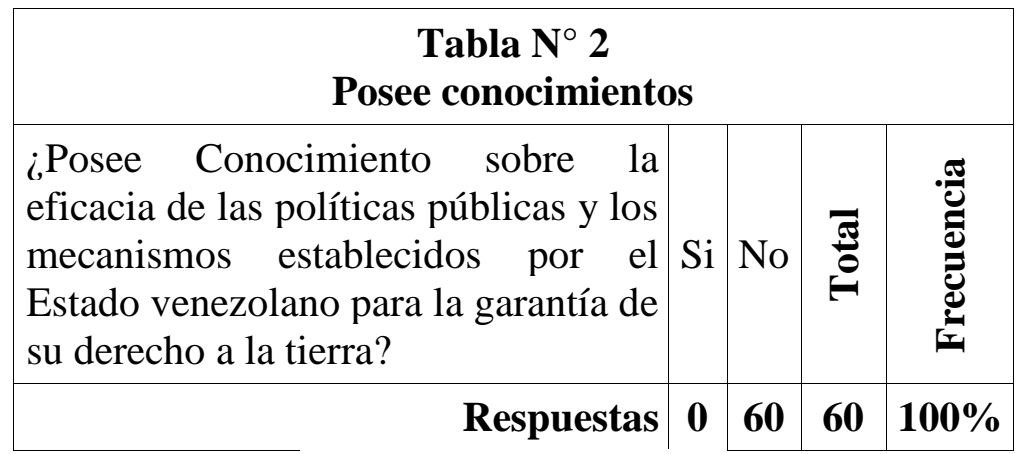

Fuente: González (2017)

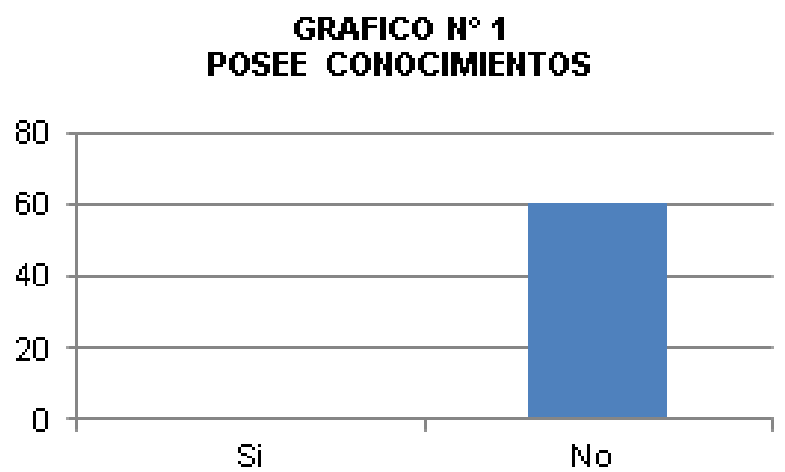

\begin{tabular}{|c|c|c|c|c|}
\hline \multicolumn{5}{|c|}{$\begin{array}{c}\text { Tabla } \mathrm{N}^{\circ} 3 \\
\text { Instituciones y programas de formación }\end{array}$} \\
\hline $\begin{array}{l}\text { ¿Cree que las instituciones gubernamentales } \\
\text { desarrollan adecuados programas de formación } \\
\text { para conocer los mecanismos establecidos por el } \\
\text { Estado venezolano para la garantía de su } \\
\text { derecho a la tierra? }\end{array}$ & $\mathrm{Si}$ & No & 恶 & 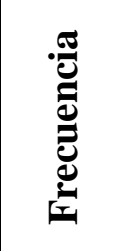 \\
\hline Respuestas & $\mathbf{0}$ & 60 & 60 & $100 \%$ \\
\hline
\end{tabular}

Fuente: González (2017)

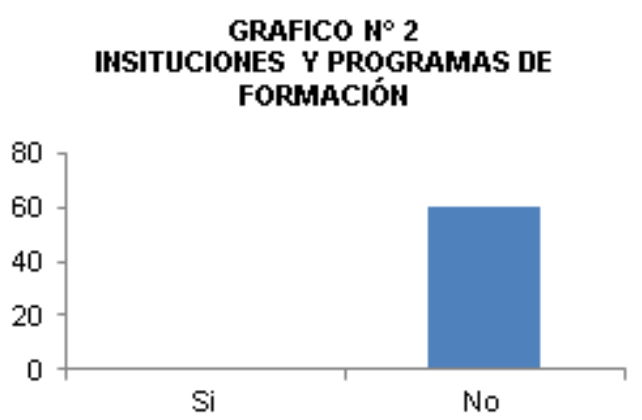


Los encuestados reconocen que instituciones gubernamentales desarrollan programas de formación, sin embargo, no son adecuados para solucionar la problemática, en sus discursos se expresan la necesidad que la comunidad vigile el cumplimiento de los derechos humanos en cada la región.

La totalidad de la población campesina encuestada coincidió que el uso inadecuado y sin los métodos establecidos para la implementación de la de programas de formación acarrea como consecuencias el desconocimiento de los derechos humanos de este grupo poblacional con necesidades especiales. Para los encuestados los derechos humanos están especialmente vinculados con el derecho a la tierra y con los derechos asociados a este como el derecho al trabajo, a la alimentación en especial dada la situación que se enfrenta la sociedad venezolana actualmente.

4.2. Describir los mecanismos y las políticas públicas del Estado venezolano para garantizar los derechos de la población campesina del municipio Paz Castillo del estado Miranda al usufructo de sus tierras.

Una vez analizadas los documentos de memoria y cuenta del Ministerio del Poder Popular para la Agricultura y Tierras podemos establecer como resultado que el Estado ha avanzado en la consolidación de una política social redistributiva del ingreso nacional. Por ello ha promulgado leyes y la consolidación de las misiones dirigidas a fortalecer el sector campesino como la Ley de tierras. Igualmente, un número importante de servidoras y servidores del Ministerio del Poder Popular de Agricultura y Tierra y sus entes adscritos, han participado en la formación para transmitir el marco conceptual de la agricultura con enfoque agroecológico y los aspectos centrales que conforman la política pública de la agricultura urbana en Venezuela, con el propósito que agricultores participen, mediante una metodología de trabajo in situ, a fin de potencializar al productor y convertirlo en un nuevo ciudadano rural, que realice un trabajo tecnificado, en sus labores diarias.

4.3. Establecer la factibilidad de un programa de formación dirigido a la población campesina del Municipio Paz Castillo del estado Miranda sobre la defensa de su derecho a la tierra.

Como se observa en la Tabla $\mathrm{N}^{\circ} 4$ y en el Grafico $N^{\circ} 3$ Factibilidad de un Programa de Formación, los resultados de la investigación arrojaron que la población campesina del Municipio Paz Castillo del estado Miranda esta población muestra un singular interés y receptividad en la posibilidad del desarrollo de una actividad educativa y formativa en materia de derechos humanos.

\begin{tabular}{|c|c|c|c|c|c|}
\hline \multicolumn{6}{|c|}{$\begin{array}{c}\text { Tabla } \mathrm{N}^{\circ} 4 \\
\text { Factibilidad de un programa de formación }\end{array}$} \\
\hline ¿Cómo Considera usted? & 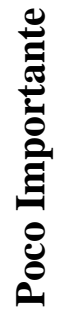 & 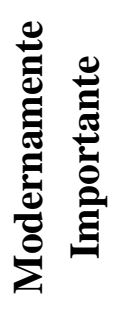 & 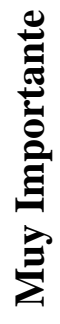 & 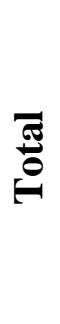 & 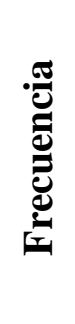 \\
\hline Participar en un Programa de Formación & & & 60 & 60 & 100 \\
\hline Sobre el derecho a la tierra & & & 60 & 60 & $\mathbf{1 0 0}$ \\
\hline En los valles del Tuy del edo. Miranda & & & 60 & 60 & 100 \\
\hline
\end{tabular}

Fuente: González (2017) 
GRÁFICO $N^{\circ} 3$

FACTIBILIDAD DE UN PROGRAMA DE FORMACIÓN

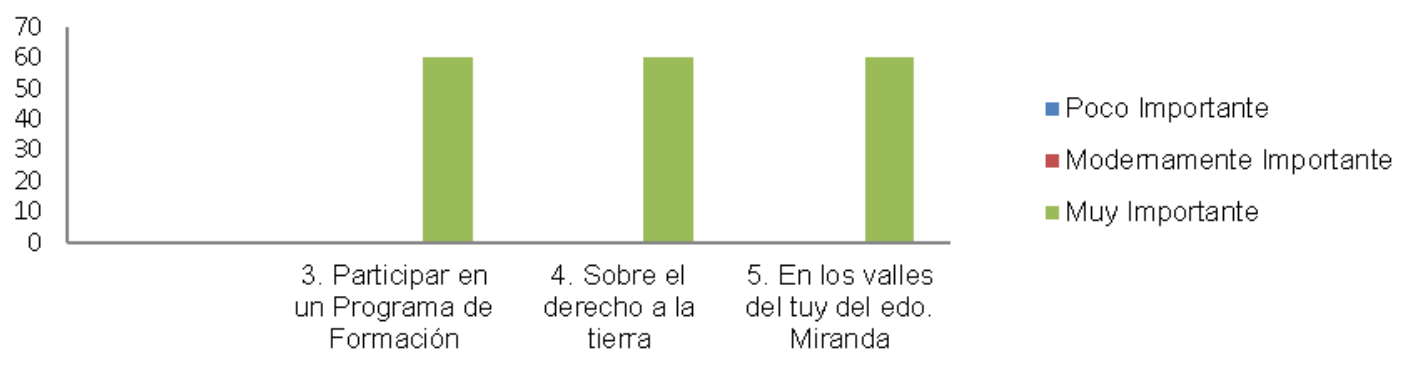

Propuesta de un Programa de Formación Educativo-Jurídico para la Sensibilización de la Población Campesina del Municipio Paz Castillo del Estado Miranda en la Defensa de su Derecho a la Tierra

\section{Presentación}

En los últimos años y como producto de la dinámica coyuntural venezolana, existe una creciente preocupación por garantizar el goce efectivo de los derechos humanos, tanto en las instituciones del sector público como del privado, lo cual ha contribuido al impulso de una cantidad significativa de movimientos y organizaciones sociales cuyo objetivo fundamental es la defensa y promoción de los derechos humanos, que Venezuela como Estado de Derecho reconoce en la Constitución; igual igual jerarquía a los tratados internacionales como a las leyes, lo cual constituye la pauta que rige todas las normas desarrolladas en el país. La defensa y promoción el Estado debe ser ejercida mediante la activación de los mecanismos establecidos para tal propósito, tal y como lo señala Brewer (2005):

(...) el derecho de acceso a la justicia, es decir, el derecho de obtener la tutela judicial de los derechos e intereses de las personas. Por tanto, la garantía genérica judicial de los derechos fundamentales es, justamente, la organización del sistema de la protección de los derechos humanos, porque su función esencial es la protección de los derechos y libertades como mecanismo eficaz y justo de protección de los derechos fundamentales (p.76).
Entre los derechos y las libertades fundamentales reconocidas por el Estado venezolano como estado social, democrático, de derecho, justicia y paz; el derecho a la tenencia de la tierra, es uno de los más controvertidos. Al respecto, Gilbert (2013) sostiene que:

(...) algunos conciben este derecho no como un privilegio, sino como un elemento fundamental en el desarrollo, por ende, debe estar al servicio de toda la población, dentro de los valores de solidaridad e igualdad de oportunidades. Sin embargo, otra corriente de pensamiento plantea que, el derecho a la tierra no suele percibirse como un problema de derechos humanos. En términos generales, el derecho a la tierra se refiere a los derechos a utilizar, controlar y transferir una parcela de tierra. (p.1)

Cabe agregar si bien es cierto, el derecho a la tierra se concibe sin tener en cuenta los derechos humanos; no obstante, es un tema esencial, ya que este derecho tiene un impacto real sobre el disfrute de otros derechos, como el de la alimentación, de la vivienda, de la salud, del trabajo, a un medio ambiente saludable y al desarrollo, entre otros. Por su parte, Ozden (2015) señala que "el proyecto de Declaración sobre los Derechos de los Campesinos que se está negociando en la ONU, se fundamenta en la reivindicación de la lucha por la función social de la tierra y la seguridad de la tenencia" (p. 24). 
En el país, históricamente ha existido gran interés por el derecho a la tierra; sin embargo, a partir del año 2001 existe una marcada tendencia, en distintos sectores, a la democratización de la tenencia de la tierra, generada por la aprobación de la Ley de Tierras. En este orden de ideas, Provea (2001) señala, en el marco de la aprobación en el año 2001 de la Ley de Tierra, que "desde la perspectiva del derecho a la tierra, la nueva Ley representa un paso adelante. Sin embargo, es posible mejorarla, sin renunciar a sus postulados básicos" (p. 23). Posteriormente, esta ley experimentó un conjunto de reformas, que incorporó una serie de mecanismos para el ejercicio, defensa y restitución del derecho a la tierra, con el propósito de profundizar los valores constitucionales de desarrollo, como estado social, democrático, de derecho, justicia y paz.

Significa entonces, que la educación cumple un rol único en el aprendizaje basado en valores, lo que crea la base para las comunidades campesinas, con fortalezas para la defensa y promoción de los derechos humanos, porque motiva un nuevo comportamiento que conduce a un cambio positivo, y promueve el diálogo con respecto a metas comunes $y$ valores compartidos necesarios para el ejercicio de los mecanismos desarrollados por el Estado, para garantizar el derecho a la tenencia de la tierra. En este contexto, el presente programa de formación educativo-jurídico tiene como propósito sensibilizar la población campesina del municipio Paz Castillo del estado Miranda en la defensa de su derecho a la tierra; en virtud que la educación en derechos humanos constituye una herramienta vital para la defensa y promoción de los derechos humanos. Al respecto, Magendzo (2002) plantea que:

(...) la educación en derechos humanos es un proceso que intenta transmitir los valores de la paz, de la tolerancia, del respeto a los derechos humanos con la intención de construir ciudadanos libres y cívicos, conocedores de cuáles son sus derechos y respetuosos con los de los demás (p. 24).
Como plantea el autor, la integración en el contenido educativo de los derechos humanos fortalece los conocimientos en esta área y responsabilidades en la sociedad. El diseño de un programa de formación facilita la introducción de la educación en derechos humanos, contribuye a transformar el modelo de aprendizaje y práctica sobre el tema. La promoción de los principios de derechos humanos, es indispensable para crear un entorno de aprendizaje que refleje y defienda los valores de los derechos humanos. Por otra parte, Laude (2000) plantea que la educación en y para los derechos humanos fomenta varios objetivos, como formar a las personas para que desarrollen la capacidad de interpretación crítica, generar cambios de actitud, fomentar actitudes de solidaridad, y cambiar el comportamiento, por medio de actividades que reflejen el respeto mutuo de las personas.

La educación constituye una herramienta que facilita la formación de ciudadanos sujetos plenos de derechos y la construcción de una ciudadanía capaz de defender y promocionar los derechos humanos. En este contexto, se ha diseñado el programa de formación dirigido a la incorporación de estrategias que contribuyan a la sensibilización de la población campesina de los Valles del Tuy, en especial del Municipio Paz Castillo del estado Miranda, mediante la educación en y para los derechos humanos, tema de una singular vigencia, dado los cambios culturales que han incidido significativamente en los valores que la actual sociedad venezolana demuestra, y la relación existente entre éstos y los derechos humanos.

\section{Justificación y fundamentación de la propuesta}

La concepción clásica de derechos humanos, sostiene que son atribuciones de la persona frente al Estado, entendiendo desde esta perspectiva, que sólo el Estado y sus

Horizontes. Revista de Investigación en Ciencias de la Educación. Vol. 1, Nro. 4, Octubre-Diciembre, 2017 
funcionarios son responsables de garantizar los derechos humanos y sólo ellos pueden constituirse en agentes de violación. En este orden de ideas, se ha diseñado el programa de formación que se presenta, el cual mejorará las competencias de los activistas de los derechos humanos, a los profesionales y técnicos miembros de las comunidades y organizaciones sociales defensoras de los derechos humanos, en su desempeño profesional.

Esta propuesta se justifica por los resultados obtenidos durante el desarrollo de la investigación, donde se evidenció que la población campesina del municipio $\mathrm{Paz}$ Castillo del estado Miranda no tiene una formación idónea para la defensa del derecho a la tierra, incidiendo esto en el goce efectivo y la activación de los mecanismos necesarios para la defensa y restitución de aquellos casos donde se vulnere este derecho; así como, los derechos sobre los cuales tiene un impacto significativo como el derecho a la alimentación, vivienda, a la salud, al trabajo, a un medio ambiente saludable, y al desarrollo, entre otros. De allí la necesidad de presentar un plan de acción con temas de formación en derechos humanos, y en especial el derecho a la tierra.

El diseño del programa de formación está sustentado en las teorías conductista, cognitivista y constructivista, en virtud que los participantes deben manejar ciertos conceptos claros de derechos humanos, y en especial el derecho a la tierra. Está conformado por cuatro (4) talleres de ocho (8) horas académicas, programados para ser realizados en dos (2) sesiones de cuatro (4) horas académicas.

Como estrategias para el desarrollo de los talleres, se consideró la experiencia de los participantes; es por ello que la metodología prevista en el programa de formación, pretende conjugar tanto el aprendizaje teórico como el práctico; por lo que se han planificado varios tipos de actividades de enseñanza centradas en los participantes, donde además de las entradas teóricas, se realizarán algunos trabajos en equipo: además se procurará una participación activa de los participantes a través de discusiones y disertaciones grupales, lo que permitirá la evaluación en su rol como participantes defensores de los derechos humanos.

\section{Objetivo de la propuesta}

\section{Objetivo General}

Formar mediante la planificación de varios talleres, a profesionales y miembros de las comunidades, población campesina y organizaciones sociales defensoras de los derechos humanos en el Municipio Paz Castillo del estado Miranda, para la sensibilización en la defensa de su derecho a la tierra.

\section{Objetivos Específicos}

1. Realizar diagnóstico sobre los conocimientos que posee la población del Municipio Paz Castillo del estado Miranda sobre derecho a la tierra y su incidencia en el goce y ejercicio de este derecho.

2. Diseñar un plan de formación dirigido a la población campesina del Municipio Paz Castillo del estado Miranda para sensibilizarlos en la defensa de su derecho a la tierra.

3. Evaluar la factibilidad y sostenibilidad de la aplicación de la propuesta en el Municipio Paz Castillo del estado Miranda

4. Desarrollar la propuesta de formación dirigido a la población campesina del Municipio Paz Castillo del estado Miranda en la defensa de su derecho a la tierra durante el segundo semestre del año 2017.

\section{CONCLUSIONES}

El presente capitulo tiene como propósito exponer las principales conclusiones que arrojó el análisis de los resultados al igual que, las recomendaciones. Los trabajos especiales de grado constituyen el espacio propicio para cualquier especialidad para la generación de conocimiento y el planteamiento de soluciones, en especial áreas tan sensibles como los derechos humanos, más aún si se 
trata del análisis al desempeño de los órganos responsables de su materialización. En este contexto, la presente tuvo como objetivo Proponer un Programa de formación educativo - jurídico para la sensibilización de la población campesina del Municipio Paz Castillo del estado Miranda en la defensa de su derecho a la tierra.

Para ello, se planteó como objetivos específicos Diagnosticar los conocimientos que tiene la población campesina del Municipio Paz Castillo del estado Miranda sobre la eficacia de las políticas públicas y los mecanismos establecidos por el Estado venezolano para la garantía de su derecho a la tierra. Describir los mecanismos y las políticas públicas del Estado venezolano para garantizar los derechos de la población campesina del municipio Paz Castillo del estado Miranda al usufructo de sus tierras. Establecer la factibilidad de un programa de formación dirigido a la población campesina del Municipio Paz Castillo del estado Miranda sobre la defensa de su derecho a la tierra. Diseñar un Programa de formación educativojurídico para la sensibilización de la población campesina del Municipio Paz Castillo del estado Miranda en la defensa de su derecho a la tierra.

Una vez analizados los resultados obtenidos se hace evidente del diagnóstico que la población campesina desconoce los mecanismos establecidos por el Estado para garantizar el derecho a la tierra, también reconocen que instituciones gubernamentales desarrollan programas de formación, sin embargo, no son adecuados para solucionar la problemática, en sus discursos se expresan la necesidad que la comunidad vigile el cumplimiento de los derechos humanos en cada la región.

En cuanto a, los mecanismos y las políticas públicas del Estado venezolano para garantizar los derechos de la población campesina del municipio Paz Castillo del estado Miranda al usufructo de sus tierras, sin embargo, el desconocimiento de los mecanismos de defensa limita el acceso a las acciones gubernamentales para garantizar el derecho a la tierra. Por otra parte, los programas de formación son diseñados desde la perspectiva de los técnicos y funcionarios institucionales muchas veces sin considerar la realidad local, especialmente de la población campesina. Es factible el desarrollo del programa dado el interés que mostro la muestra de la población campesina objeto de estudio, producto de la situación que atraviesa la población campesina especialmente en el estado Miranda.

\section{REFERENCIAS}

Amnistía Internacional. (2012). Objetivos de la educación en derechos humanos. Amnistía [Revista en Línea]. Extraído el 2 de abril de 2017 desde http://www.amnistia.ong

Bolívar, L. (2001) Significados Teóricos y prácticos que tiene para la educación en derechos humanos en ll contexto de los países latinoamericanos. [En Línea]. Extraído el 2 de abril de 2017 desde http://www.derechos.org.ve.

Brewer, C. A. (2005). Mecanismos nacionales de protección de los derechos humanos (garantías judiciales de los derechos humanos en el derecho constitucional comparado latinoamericano). [En Línea]. Extraído el 2 de abril de 2017 desde https://www.iidh.ed.cr

Casal, J. (2006). Los derechos humanos y su protección. Caracas, Venezuela: UCAB.

Comisión Interamericana de Derechos Humanos (2008). Lineamientos para la elaboración de indicadores de progreso en materia de derechos económicos, sociales y culturales. [En Línea]. Extraído el 03 de abril de 2017 desde http://www.cidh.org.

Comité Asesor Consejo de Derechos Humanos (2012). Estudio definitivo del comité asesor del consejo de derechos humanos sobre la promoción de los derechos de los agricultores y de otras personas que trabajan en las zonas rurales. [En Línea]. Extraído el 03 de abril de 2017 desde http://www2.ohchr.org

Constitución de la República Bolivariana de Venezuela. Publicada en Gaceta Oficial del jueves 30 de diciembre de 1999, $\mathrm{N}^{\circ}$ 36.860 . 
Constitución de la República Bolivariana de Venezuela (1999) Publicada en: Gaceta Oficial de la República Bolivariana de Venezuela №. 5.453 (Extraordinaria) de fecha marzo 24 del 2000.

Cunill, N. (1991). Participación dilemas y perspectivas para la democracia de los estados latinoamericanos. Caracas, Venezuela: CLAD.

Cunill, N. (1995). La rearticulación de las relaciones estado-sociedad en búsqueda de nuevos espacios en: reforma y democracia: Caracas, Venezuela: CLAD

De Nóbrega, J. (2017). Normas EDEHU. Especialización en derechos humanos. Dirección de investigaciones y postgrado. Universidad Nacional Abierta. [En Línea]. Extraído el 03 de abril de 2017 desde en: https://drive.google.com/file/d/0B8BiCo xC60Q1eWV2Ulc5eGRrYXM/view

Delahaye, 0. (2003). La privatización de la tierra agrícola en Venezuela, desde Cristóbal Colón: la titulación (14922001). [En Línea]. Extraído el 03 de abril de 2017 desde http://www.saber.ula.ve

Diario el Nacional (2016). En Valles del Tuy hay 52,5\% más homicidios que en 2014 . Extraído el 29 de marzo de 2017 desde www.el-nacional.com.

Edelman, M. (2013). Los derechos de los y las campesinas en las Naciones Unidas. [En Línea]. Extraído el 03 de abril de 2017 desde http://www.hunter.cuny.edu

F.A.O. (2015). Qué es el acceso a la tierra. [En Línea]. Extraído el 29 de marzo de 2017 desde http://www.fao.org

Fernández, A. (2004). Universidad y currículum en Venezuela. Hacia el tercer milenio. Caracas: UCV.

Food and Agricultural Organisation of The United Nations (FAO). 2002. Land tenure and rural development. Rome. (FAO Land Tenure Studies, 3) [En Línea]. Extraído el 03 de abril de 2017 desde http://www.fao.org

Freire, P. (1971). La educación como práctica de la libertad. 3a edición. Montevideo: Editorial Tierra Nueva.

Gil, J.A., Nain, M. A. Romero, A. Stambouli y D.B. Urbaneja (1979). Introducción al análisis de las políticas públicas en Venezuela. Caracas: CONICIT.

Gilbert, J. (2013). Derecho a la Tierra como Derecho Humano: Argumentos a Favor de un Derecho Específico a la Tierra. Sur Revista Internacional de Derechos Humanos $N^{\circ} 18$ noviembre 2013 [Revista en línea]. Extraído el 2 de abril de 2017 http://www.corteidh.or.cr

Jones, Ch. O. (1984). Una introducción para el estudio de las políticas públicas. México: Brooks \& Cole Publishing.

Kelly Janet (2003). Políticas públicas en América latina - teoría y práctica. Caracas: IESA.

Laude, R. (2000). Educación Popular en Derechos Humanos: 24 guías de actividades participativas para maestros y facilitadores. [En Línea]. Extraído el 2 de abril de 2017 desde http://www.iidh.ed.cr

Ley de los Consejos Comunales. Gaceta Oficial № 5.806 (Extraordinaria) de fecha $10 \mathrm{de}$ abril del 2006.

Ley de Tierras y Desarrollo Agrario. Gaceta Oficial de la República Bolivariana de Venezuela $\mathrm{N}^{\circ} 37323$ del 13 de Noviembre de 2001.

Ley de Tierras y Desarrollo Agrario. Gaceta Oficial № 5.771 (Extraordinaria) de fecha 18 de Mayo del 2005.

Machado M, J. (2008). Estudios de los consejos comunales en Venezuela. Caracas: Fundación Centro Gumilla.

Magendzo, A. (2002). Los derechos humanos. Un objetivo transversal del currículum. [En Línea]. Recuperado el 2 de abril de 2017 en http://www.iidh.ed.cr

Moreno Olivos, Tiburcio. (2012). La evaluación de competencias en educación. Sinéctica, (39), 01-20. [En Línea]. Recuperado el 30 de abril de 2017, en: http://www.scielo.org

Moriarty, K. (2004). Crear ciudadanos activos en el campo de los derechos humanos: El papel de la educación en derechos humanos dentro de Amnistía Internacional. Publicado en: Tarbiya. Revista de Investigación e innovación educativa. [Revista en Línea]. Recuperado 
el 2 de abril de 2017 en http://www.amnistiacatalunya.org

Mujica, R. (2001). La metodología de la educación en derechos humanos. [En Línea]. Extraído el 2 de abril de 2017 desde http://www.iidh.ed.cr

Núñez, Jesús. (2004). Los saberes campesinos: Implicaciones para una educación rural. [Revista en Línea]. Extraído el 03 de abril de 2017 desde http://www.scielo.org.

Ozden, M. (2015). El derecho a la tierra. [En Línea]. Extraído el 03 de abril de 2017 desde http://www.alainet.org

Parsons Wayne (2007). Políticas públicas: una introducción a la teoría y la práctica del análisis de políticas públicas. México FLACSO.

Pearse, A. y Stiefel, M. (1980). Participación popular: un enfoque de investigación. [En Línea]. Extraído el 03 de abril de 2017 Recuperado en http://www.revistainvi.uchile.cl

Pérez, C. (2009). La educación no formal desde el método de Paulo Freire. [En Línea]. Extraído el 2 de abril de 2017 Recuperado en: http://secviccentarticulo.blogspot.com

Pizani, M. (2009). La participación comunitaria como ejercicio de control social. Caracas, Venezuela: UCAB

Ramos, Z y De Nobrega, J. (2017). Guía de estudio para la elaboración del proyecto especial de grado. [En Línea]. Extraído el 29 de marzo de 2017 Recuperado en https://drive.google.com/file/d/0B8BiCo xC60Q1RFI2ZWdzVHk0V2s/view

Rojas C, P. (2007). El papel de la educación no formal en el desarrollo rural: análisis de la incidencia del programa de multiplicadoras de salud y bienestar rural, en la comunidad beneficiada del municipio de Líbano, Tolima. [En Línea]. Extraído el 2 de abril de 2017 desde http://www.javeriana.edu.co

Salvador, E. Y Pacheco-Vega, R. (2009). Sistemas y Políticas de Investigación, Desarrollo E Innovación. Algunas Propuestas. [En Línea]. Extraído el 2 de abril de 2017 desde http://www.scielo.org.mx

Torre, M. (2000). El ciudadano democrático. Utopía sensata de la posmodernidad educativa. [Revista en Línea]. Extraído el 2 de abril de 2017 desde http://redie.ens.uabc.mx

Torres, M. (2009). Tipos de educación (Formal, No Formal e Informal) Extraído el 2 de abril de 2017 desde: http://edurecblog.com

Trilla, J. et al. (2003). La educación fuera de la escuela. Ámbitos no formales y educación social. [En Línea]. Extraído el 2 de abril de 2017 desde http://www.ehu.eus

Tunnermann B, C.: Educación en derechos humanos en los sistemas educativos. [En Línea]. Extraído el 2 de abril de 2017 desde http://www.iidh.ed.cr

UNESCO. (1974). Recomendación sobre la educación para la comprensión, la cooperación y la paz internacionales, y a la educación relativa a los derechos humanos y las libertades fundamentales. [En Línea]. Extraído el 2 de abril de 2017 desde http://unesdoc.unesco.org

UPEL (2016). Manual de trabajos de grados de especialización, maestría y tesis doctorales. Caracas, Venezuela: UPE 